\title{
Was there a Big Bang?
}

Eurasian Center for Big History \& System Forecasting, Oriental Institute, Russian Academy of Sciences

\begin{abstract}
The idea that our Universe emerged as a result of the extraordinary power of the Big Bang from singularity (i.e., a state of an infinitely small quantity and infinitely high concentration of matter) is still very popular today. It was one of the main postulates of the Big Bang theory that completely formed in the 1960s-1970s. However, at present this idea as well as the Big Bang theory is outdated, although it is still shared by many scientists. Being widespread since the end of the 1970s the Inflation theory appears more modern. The main reason for the emergence of the Inflation theory was that the Big Bang theory could not satisfactorily explain a number of the contemporary parameters of the Universe.

The Inflation theory makes still widespread views of the Big Bang theory archaic, in particular as regards the following points: 1) the history of the Universe started with the Big Bang; 2) it started with the singularity. According to the Inflation theory, the Big Bang was not the beginning and the moment of the origin of the Universe, but it was preceded by at least two epochs: inflation and post-inflationary heating. That is, the Big Bang or precisely the hot Big Bang is just a phase transition from the state of cold inflation to the hot phase. Since the Inflation theory does not consider the Big Bang as the initial phase there emerges an intricate problem of the role of the Big Bang in the process of the formation the Universe as a whole. The paper considers the confusion with the Big Bang notion, a number and sequence of 'bangs' and why the theory can dispense easily without the notion the Big Bang. We will also discuss some advantages and disadvantages of the Inflation theory.
\end{abstract}

\section{Key Words}

Big Bang, hot Big Bang, inflation, Universe, inflaton, false vacuum, the Inflation theory, singularity, quantum fluctuation, post-inflationary heating.

Correspondence | Leonid Grinin, Леонид Гринин <leonid.grinin@gmail.com>

Citation | Grinin, L. (2018) Was There a Big Bang? Journal of Big History, III(1); 51 - 59.

DOI | http://dx.doi.org/10.22339/jbh.v3i1.3130

\section{ntroduction: Big Bang Theory's Limitations
and the Emergence of Inflation Theory}

It is supposed that our Universe emerged about 13.82 billion years ago from the unknown state and substance. The history of the Universe, particularly its initial stages, is a scientific reconstruction whose most points are still considered to be scientific hypotheses. The latter sometimes may seem unbelievable ones. It is not surprising that there is no agreement among physicists and cosmologists about many issues of the initial stages of the history of the Universe. Completely formed in the 1960s-1970s the Big Bang theory has been popular long enough (about history of the term 'Big Bang' see Wood 2018: 1-4). At present it is outdated in its certain aspects, although it is still shared by many scientists. Being widespread since the late 1970s the Inflation theory appears more modern.

The main reason for the emergence of the Inflation theory was that the Big Bang theory could not satisfactorily explain a number of the contemporary parameters of the Universe, in particular why the 
Universe is so homogeneous, isotropic, 'large' (spatially flat) and hot (Gorbunov and Rubakov 2010: 341; Guth 1997, 2002, 2004).

Due to the emergence of the Inflation theory many problems of the Big Bang theory can be eliminated. The Inflation theory makes still widespread views of the Big Bang theory archaic. For our topic the following points of this theory are more important: 1) the history of the Universe started with the Big Bang; 2) it started with the singularity (i.e., a state of an infinitely small quantity and infinitely high concentration of matter). Below we will consider the main ideas of the Inflation theory. At present one should note that according to this theory the Big Bang was not the beginning and the moment of the origin of the Universe, but it was preceded by at least two epochs: inflation and postinflationary heating. That is, the Big Bang or precisely the hot Big Bang is just a phase transition from the state of cold inflation to the hot phase. Furthermore, due to the fact that the Inflation theory does not regard the Big Bang as the initial phase there emerges an intricate problem of the role of the hot Big Bang as a whole. In the words of Alan Guth, it is not explained what 'exploded', how it 'exploded' and what caused the 'explosion' (Guth 1997). Thus, it is not surprising that (as we will see below) as a rule there is no clear description of the phase of the Big Bang in the modern research.

In this introduction we find it is necessary to give a detailed explanation of our goals with respect to important subject of this article.

1. The article is written for the Big Historians and the Big History proponents. For them the article may be important since it allows defining important peculiarities that may often escape attention. With respect to the physical theory there will be nothing new for astronomers and astrophysicists, but in terms of the evolutionary theory and philosophy they may find something interesting.

2. The article is devoted to the analysis of whether one can consider the Big Bang as a certain real phenomenon or such a view is just a heritage of what was previously established in science. I am convinced that Big Bang is nothing more but a metaphor (just as Barry Wood [2018] and other scientist [see below]).

3. I would like to point out the aspects of the inflation theory which often escape attention. If one cannot speak about the Big Bang as a real event then it is important to define in what way one should interpret this notion within the inflation theory framework. Among the proponents of the inflation theory there exist disunity and different approaches often slightly manifested. I consider any clarification of this problem to be of vital importance for the development of Big History as a discipline and as a subject taught. And I think they provide insight into these complicated issues.

It is worth adding that when I define the Big Bang theory as outdated I mean in the first place its classical form, i.e. the Big Bang theory of the 1970s which is still popular. It is just like saying that classical Newtonian mechanics became outdated after the elaboration of Einstein's theory. It is really so. But at the same time under certain conditions it is valid and is incorporated as an individual case in the theory of relativity. The same happens with the Big Bang theory.

So today the Big Bang theory is firmly integrated within the inflation theory ${ }^{1}$. Thus, on the whole, it is probably unreasonable to speak about a dichotomy between the two theories which are mostly unified today. However with respect to the issue under study, they significantly diverge since the place of the Big Bang within the modern cosmological theory is unclear and undefined. While recognizing that the inflation theory forms a comprehensive theory with Big Bang theory, at the same time I argue that their merging has generated a number of problems and actually provokes an internal contradiction within the

1 It is possible to meet both statements: that the inflation theory incorporates the Big Bang theory and the opposite view that it is the inflation theory that is incorporated into the Big Bang theory. I prefer the former approach but actually, it is not so important how to express this idea since the two theories have actually merged into one. Nevertheless, these discrepancies just show that there is much confusion about the big bangs (which I tried to show in the article). 
inflation theory itself. And that is what I will try to prove in what follows.

\section{Main Ideas of Inflation Theories}

Historically, Alexei Starobinsky's model of inflation (1979) was the first model developed in detail. But the best-known Inflation theory was first formulated in the famous article by Alan Guth in 1981, which continues to promote it extensively. Within the framework of this theory 'the fundamental properties of our Universe (i.e., it is homogeneous, isotropic, spatially flat and hot) appear as the consequences of extremely unnatural initial conditions' (Gorbunov and Rubakov 2010: 341).

The Universe before the hot Big Bang. The inflationary stage. According to the Inflation theory our Universe's origin was the result of quantum fluctuation (i.e., negligible small fluctuation but still having certain spatial parameters ${ }^{2}$ ). This fluctuation has put the forces of the so-called false vacuum in motion. A false vacuum is a hypothetical state of matter, in which, matter is repulsed and space is expanded due to negative pressure. That is why this stage is called the inflationary stage (i.e., inflation of the Universe). The Universe has reached enormous proportions in the smallest fractions of the second. One should mention that a false vacuum had constant temperature. That is why the inflation is defined as cold. The heating had begun due to the processes described below. Vacuumlike energy as well as false vacuum itself is now often called the inflaton.

Completion of the inflationary stage, postinflationary heating. The false vacuum is an unstable state of matter, so it started to decay quickly. On the whole, the inflationary period (as well as all the initial stages of the early Universe) was very short. Nevertheless it is important for the theory that it must not be smaller than an extremely short period of time, measured in the smallest units, so-called Planck units (from 70 to 100 such units within the smallest fraction

2 It differs much from the Big Bang theory which regards the starting point of the Universe with the singularity (see above). of a second) $)^{3}$. This duration in terms of the Inflation theory was called the slow-roll of a scalar (inflaton) field. During this process the potential energy of this field decreased, transforming into the kinetic one. It is supposed that this leads to the formation of the socalled boson condensate. Eventually, the potential energy of the inflaton (inflaton field) reaches a minimum at a certain moment. This means that the conditions necessary for exponential increase are violated and the inflationary stage ends.

Thus, this leads to a rather rapid heating of the Universe. There comes the stage of post-inflationary heating, in which the boson condensate decays due to the vibrations (oscillations) of the inflaton field, which has reached its minimum energy. During the oscillation of the inflaton field one can observe the beginning of the formation of different particles about the nature of which there are different assumptions. The energy of the inflaton transforms into the energy of the emerging particles as a result of their interaction with the rapidly changing inflaton field. Figuratively speaking, one can observe 'pumping out' of the energy that led to the rapid heating of the Universe and the formation of elementary particles of ordinary matter. In other words, the entropy that was previously low in the false vacuum increased sharply. At the same time there was a rapid expansion of the Universe. And the inflationary equation of state of matter transforms into the powdered one. And later, when the heating had reached its peak, the powdered equation of state transformed into a radiation dominated equation. In other words, when reaching an ultrahigh temperature, the matter passed into the state of 'super-hot plasma consisting of free quarks, gluons, leptons and highenergy quanta of electromagnetic radiation' (Levin 2010 $)^{4}$. Hence, within a fraction of a second there took place successively equations of state of a false vacuum

3100 Planck times is something like a period of time from $5 \times$ $10^{-44}-5 \times 10^{-42} \mathrm{~s}$.

4 An ordinary matter that had appeared as a result of boiled vacuum and then a hot 'bang' had been remaining in a state of hot plasma for hundreds of thousands years (until the process of hydrogen recombination). 
- powdered - radiation dominant (for more details see Grinin 2013).

Was there the hot Big Bang Phase? There are discussions about the temperature which the postinflation Universe achieves as a result of these processes. In any case, it was very high, although, most likely, it was lower than it was expected in the Big Bang theory 5 . According to cosmologists and physicists, this leads to a kind of 'boiling up' of the vacuum, which, it should be noted, has already occupied plenty of space by that time. Postnov (2001) notes that during the period of $10^{-34} \mathrm{~s}$. the stage of inflation 'prepares' the primary very hot substance in a very small area, and it expands by inertia.

Thus, in the previous description one can see a phase transition from the state of cold inflation to the hot phase. Just in this point of the Inflation theory there emerges a problem of identification of the place, role, and even reality of the Big Bang. The Inflation theory does not give any definition of this concept. On the one hand, they maintain that heating had resulted in a hot Big Bang which further dispersed the expansion of the Universe. One cannot find any great explosion in the hot Big Bang phase unlike the picture drawn in the classical Big Bang's scenario. Of course, one can call heating of the Universe the Big Bang, but the heating is a process, it was not momentary as a sudden explosion assumed by the Big Bang theory. We will return to the problem of the Big Bang concept below.

\section{Comments on the Inflation Theory}

The advantages of the Inflation theory from a philosophical point of view in comparison with the Big Bang theory are as follows: 1) the existence of matter and the Universe before the phase of inflation is supposed; 2) anyway, the process of the formation of the Universe looks exactly like a process (although very fast), but not as an act of creation from nothing; 3) the original size of the Universe, although small,

5 Although there are no direct experimental indications that there were temperatures above several $\mathrm{MeV}$ in the Universe (i.e., several tens of billions of degrees) (Gorbunov and Rubakov 2012). but it is still more verisimilar than the singularity (the latter is an artifact of outdated cosmology); 4) the introduced hypothetical substance - the inflaton field - explains the processes as a whole with the help of physics, and not simply by the assumption of an explosion.

In the Big Bang theory, as the beginning of everything which emerged from the singularity, it was believed that the classical space-time started to form immediately in the course of explosion, because the Universe began to expand at once after the hypothetical state of singularity and also acquire the related characteristics. As Hawking (2001) wrote, Einstein's general relativity theory concludes that space-time arose at the singular point of the Big Bang. However, we proceed from the fact that the hot Big Bang was preceded by the inflationary phase, during which the Universe significantly expanded. Thus, a very rapid expansion of the Universe during the given period leads to the origin of classical space and time.

\section{Disadvantages of the Inflation theory and its Physical fatalism}

Now let us consider the disadvantages of the Inflation theory. They are as follows: 1) Introduction of the hypothetical substance. Inflation requires the introduction of some powerful repulsive force for its explanation (i.e., the inflaton field or a false vacuum with negative pressure), the nature of which is not clear in many respects (see May et al. 2007: 38-39). In the inflaton field the laws of ordinary gravitational physics change, because 'matter becomes not a source of attraction, but a source of repulsion' (Sazhin 2002: 38).

Filling gaps with hypothetical kinds of matter is a form of science development. In this case it seems as a too bold idea.

2) The assumption that initially the Universe had very small (almost Planck) size and a huge Universe could arise from that size. We have no example of evolutionary processes when something very large would have turned out from one tiny unit. The process always proceeds as either the coexistence 
of the mass of small units, which then form a new macrosubstance (system), or the gradual acquisition by a certain number of small units of the ability to grow and, as a result, the emergence of large units. ${ }^{6}$ 3) The Universe's origin time is too short. In the end, although the Inflation theory significantly withdrew from the concept of the 'act of creation', as opposed to the Big Bang theory, but the generic features of this approach are still visible. 4) As in the case with the Big Bang theory an issue about the origin of the Universe which appeared to be 'almost from nothing' raises many questions (Rubin 2004). It is also unclear, 'where does the material come from in the first state of the world' (Cherepashchuk and Chernin 2004: 278)7 .

5) In general, both the Big Bang theory and the Inflation theory proceed from the fact that they must explain the present observed states of the Universe, including Hubble's law, the spatial homogeneity of the Universe, its flatness, etc. How far is such predeterminancy possible in terms of evolution? ${ }^{8}$

Why should these states be explained by the very initial conditions? Why could not they arise later under the influence of any factors? Apparently, this is connected both with the desire of cosmologists and physicists to see a complete picture that would explain everything, and that otherwise, if the theory of

6 To a certain extent the assumption of multiple of multi-faceted universes also implies such a variant of gaining the ability to grow, but the idea of multitude of universes is too speculative to be associated with evolutionary processes.

7 Postnov (2001) points out that the exponential growth in the sizes of the area with constant density means the growth of mass (energy) inside the area 'out of nothing', which might seem strange at first sight. However, there is no violation of energy conservation law - the growth of the positive energy is exactly compensated by the negative energy of the gravity field, which is created by the 'emerging' positive energy inside the expanding area. Therefore, the total energy remains the same in the course of inflationary expansion (see also Sazhin 2002).

8 Even the proponents of such views have to admit that 'according to modern ideas, space-time in the Planck scale is a fantastic figure, more like a monster from horror films than the object of physical research. Future research will show whether this picture is correct' (Sazhin 2002: 81). About evolutionary approaches in respect of Cosmic phase of the Big History see Grinin 2014; 2018. the origin of the Universe does not explain the present observed circumstances, then it is easily refuted and, in fact, not even considered. As a result, the emergence of Hubble's law is included in the Inflation theory, although why should not this expansion (if the redshift would not be later explained in another way) emerge later? The expansion of the Universe having arisen at the very first moment does not change by inertia. It looks rather fatalistic ${ }^{9}$. Moreover, the entire subsequent large-scale structure of the Universe was therefore predetermined by the smallest density fluctuations, which already appeared at the inflationary stage within extremely short fractions of milliseconds. It is very sad to realize that everything was decided in such a short period and in such a small amount (from the Planck size to $1 \mathrm{~cm}^{3}$ ) of matter. Although the Inflation theory aims to withdraw from the concept of singularity with its full uncertainty in the physical realm, nevertheless the original dimensions are difficult to perceive.

One should mention that quantum dimensions of the original Universe in comparison with the singularity from the point of view of physics is a principally different state, since it allows using already known or at least formulated hypothetical laws and forces. But from the perspective of the ideas of evolution the differences between singularity and quantum dimensions are not considerable.

\section{Confusion and Problems with Big Bangs}

As we have seen from the above discussion, the stage of the hot Big Bang succeeded the postinflationary heating stage. However, there are still a number of scientists who, just as before, consider the Big Bang as the moment of the origin of the Universe followed by inflation. However, this disagreement can be explained not only by differences in points of view but also by the confusion in terminology. The question is that when speaking about the Big Bang as an event preceding the beginning of inflation, it is often meant not the hot Big Bang (i.e., classical Big Bang), but

9 Not to mention the fact that this contradicts the fact according to which the speed of the receding galaxies not only decreases but increases. 
another one, i.e. the pre-inflation Big Bang.

Thus, today speaking about the Big Bang, it is necessary to specify which explosion is mentioned. The fact is that there is no common terminology concerning the Big Bang in physics and cosmology, which study the early Universe: there is considerable confusion here.

Sometimes the followers of the Inflation theory mention the Bang that preceded the inflation stage. They might regard this bang as above mentioned quantum fluctuation or another hypothetical event of uncertain origin. Sometimes they talk about such Big Bang as a special phase of early history of the Universe. Unfortunately they do not clarify whether this Big Bang was the trigger for the quantum fluctuation, or it is just the beginning of the inflationary stage. In any case this Big Bang was definitely cold but not hot. However, some researchers do not identify the preinflation cold Big Bang or do not mark it out as a special stage because such a variety in approaches implicitly creates a great confusion in our understanding of the notion of the Big Bang. Were there two Big Bangs or was only one or none at all? And after what stage it occurred? The confusion is growing because the Big Bang theory also implies the inflationary stage. But the sequence of stages differs from that of the Inflation theory. According to the Big Bang theory, the Big Bang was the first to occur and led to great inflation. And the Inflation theory suggests that the hot Big Bang resulted from the inflation. As we will see below, such a shift of the processes' order makes the Big Bang unnecessary stage in the sequence of events that occurred in the Universe. One should also note that not all researchers distinguish the stage of postinflationary heating.

As a result this situation seems paradoxical. On the one hand, practically, there are no scientists who would definitely reject the Big Bang. On the other hand, a number of researchers who use this concept as something conventional, but indefinite, increases. It appears that implicitly or even explicitly they understand that the theory can easily avoid using the
Big Bang notion. However, because the direct negation of the Big Bang may cause difficulties, probably they think that the best way to avoid problems is the indistinct mentioning of this moment. Thus, one should mention that the Big Bang seems to become a kind of metaphor, an indicator of fidelity to the mainstream, playing a role similar to that of the incomprehensible god in deism philosophy ${ }^{10}$. We recall that the situation is greatly complicated.

Among many followers of the Inflation theory there is an implicit assumption that there could be two trigger events which can be described as 'bangs', one of which preceded the inflation, and the other followed it.

But the description and characteristics of the preinflation Big Bang are even more obscure than those of the hot Big Bang. It also does not have any common term; there are references to the Planck era of the Big Bang, the early Big Bang stage, the real Big Bang, etc. One should mention that due to this terminological and theoretical confusion it is extremely difficult to understand whether one or two explosions are meant, as well as to describe the real sequence of stages ${ }^{11}$. If there were two Big Bangs then the origin of the Universe would schematically look like this: the preinflationary Big Bang - inflation (expansion of the Universe) - post-inflationary heating of the Universe - the hot Big Bang. But such a reconstruction is not presented anywhere because perhaps as was mentioned above it is easier to avoid difficulties. Most commonly mentioning of the Big Bang among physicists simply looks like a tribute to a tradition they dare not to violate, and therefore such mentions are rather ritual than filled with specific content ${ }^{12}$. In general, it appears that

10

11 It is difficult to understand also from Guth's article with the title 'Was Cosmic Inflation the 'Bang' of the Big Bang?' which is relative to our topic.

12 Though one can find the following arguments. It is shown that such an event as the hot Big Bang is not a necessary stage in the Inflation theory. Now it is clear that the inflationary stage played a role of the 'bang' (Postnov 2001). The moment when the Universe is heated up is now called the Big Bang (Ibid.). The boundaries between inflating and thermalized 
the early history of the Universe may well do without using the concept of the Big Bang, using the scheme: fluctuation (whatever it may have been caused by) inflation - post-inflationary heating.

Thus, among a large number of astrophysicists the very idea of the Big Bang has been losing not only its substantiality and uniqueness, but the need in general. However, at the same time among others and especially among those who popularizes the early history of the Universe one can observe dominating desire to see something extremely real and apocalyptic in the Big Bang. Perhaps, it would be too strong to call the Big Bang 'a misleading, ugly and trivializing name'. (as Timothy Ferris did; see Wood 2018: 2). Nevertheless in the light of modern points of view it is very necessary to regard the Big Bang not as a real huge explosion but rather as a metaphor that still exists due to tradition ${ }^{13}$. The matter is that 'the Big Bang, just as we imagined it traditionally, most likely did not occur at all' (Mukhanov and Orlova 2006). At the same time, most initial conditions that determine the most important characteristics of the modern Universe are also referred to the inflationary stage, rather than the hot Big Bang.

\section{Conclusion}

The importance of the Inflation theory. The theory of the Big Bang could not explain very much,

regions play the role of the Big Bang for the corresponding thermalized regions (Garriga and Vilenkin 2001; Vilenkin 2006, 2010; in all cases, emphasis added by me. - L. G.).

13 Perhaps, it also has some sense from pedagogical point of view. In the paper by Wood (2018) one can see a discussion on possibilities of using the notion of Big Bang for pedagogical and other purposes as well as the author's suggestion to use as synonym of 'Big Bang' term 'the big beginning ' as beginning of TIME, SPACE, MATTER and ENERGY as well as other initial substances. He clarifies his goal: 'Assuming "big beginning" as a non-contentious synonym for big bang, the task of communication must be redefined: How can this incomprehensible event when time began, space unfolded, matter appeared, and energy bifurcated into various forces be formulated as imaginative narratives that will broaden and deepen its meaning and significance in harmony with discoveries over the past half century?' (Ibid.: 3). which could be explained precisely with the help of the Inflation theory. At present, the Big Bang theory is firmly integrated with the Inflation theory. From the point of view of cosmology and physics the introduction of the stages preceding the hot Big Bang more or less successfully solves all the problems related to the initial data of the hot Big Bang epoch, and eventually explains the flatness, homogeneity and isotropy of the observed Universe. The inflationary era is very important for modern cosmological and cosmophysical concepts. Alan Guth explains with enthusiasm, 'Inflation is not just a theory of the initial (ultimate) beginning, but it is a theory of evolution that explains essentially everything that we see around us, starting from almost nothing' (Guth 2002; about creation from nothing see our comments above).

However, one should understand that the emergence of the Inflation theory is the result of searching for such physical conditions under which it would be possible to explain the characteristics of the modern Universe. For modelling such initial conditions some scientists introduced hypothetical states of matter and energy. Therefore, it is absolutely normal that there are dozens of competing models of the inflationary stage, as well as the fact that nearly all parameters of this stage are unclear. What is really surprising is that science can put forward well-structured and reasonable hypotheses about such distant and extremely short periods.

Thus, on the one hand, the Inflation theory is a triumph of possibilities of modern cosmology and physics, but on the other hand, it perfectly demonstrates the limits of our knowledge, and the extent to which these hypotheses can be exotic and strange to explain things near these limits.

One should not forget that in these cases we are talking not about even theories but paradigms (Guth 1997), not the proved facts but hypothetical events and substances. It is also worth agreeing with Guth's (1997) statement that if it is true that the Universe arose from inflation, we cannot regard the quantum fluctuation as the cosmic origins. This idea leads us closer to its beginning. However, it is absolutely unclear, whether there was something before the inflation and what it 
was. There are a number of theories about these topics.

In particular there is a wide variety of very original theories according to which our Universe is not the only one, but just one of the myriad universes of the Multiverse ${ }^{14}$. According to some theories, these universes do not contact with each other, according to others the collisions of universes cause Big Bangs. Anyway, according to such approaches, the origin of our Universe 13.82 billion years ago is an ordinary event in Multiverse. However it is a very important event for humanity and the starting point for Big History because any history must have a beginning.

14 E.g., according to A. Linde's theory, 'the area of the Universe that we are bserving now occupies a part of one of these "bloated" clusters' (Rubakov and Gorbunov 2010: 357).

\section{References}

CherepashchyukA. M., and Chernin A. D. 2004. The Universe, Life, Black Holes (Science for Everybody). Fryazino: Vek 2. In Russian (Черепащук А. М., Чернин А. Д. 2004. Вселенная, жизнь, черные дыры (наука для всех). Фрязино: Век 2).

Garriga J., and Vilenkin A. 2001. Many Worlds in One. URL: http://cds.cern.ch/record/486136/files/0102010.pdf

Gorbunov D. S., and Rubakov S. A. 2010. Introduction to the Theory of the Early Universe. Cosmological Perturbations and Inflationary Theory. Moscow: URSS. In Russian (Горбунов Д. С., Рубаков С. А. Введение в теорию ранней Вселенной. Космологические возмущения. Инфляционная теория. М.: УРСС).

Gorbunov D. S., and Rubakov S. A. 2012. Introduction to the Theory of the Early Universe. The Theory of Big Bang. Moscow: LKI. In Russian (Горбунов Д. С., Рубаков С. А. Введение в теорию ранней Вселенной. Теория горячего Большого взрыва. 2-е изд. М.: ЛКИ).

Grinin L. E. 2013. Big History of the World Development: Cosmic Evolution. Volgograd: Uchitel. In Russian (Гринин Л. Е. 2013. Большая история развития мира: Космическая эволюция. Волгоград: Учитель).

Grinin L. E. 2014. The Star-Galaxy Era of Big History in the Light of Universal Evolutionary Principles. Teaching \& Researching Big History: Exploring a New Scholarly Field / Edited by Leonid E. Grinin, David Baker, Esther Quaedackers, and Andrey V. Korotayev. - Volgograd: 'Uchitel' Publishing House. Pp. 163-187.

Grinin L. E. 2018. Evolution of the Early Solar System in Terms of Big History and Universal Evolution. Journal of Big History Volume II Number 1 Spring 2018 http://dx.doi. org/10.22339/jbh.v2i1.2252Pp. 15-26

Guth A. H. 1997. Was Cosmic Inflation the 'Bang' of the Big Bang? Beem Line 27(3). URL: http://ned.ipac.caltech.edu/ level5/Guth/Guth1.html

Guth A. 2002. The Inflationary Universe. URL: http://www. edge.org/conversation/the-inflationary-universe-alan-guth

Guth A. 2004. Inflation. Carnegie Observatories Astrophysics. Series 2: Measuring and Modeling the Universe / Ed. by W. L. Freedman. Cambridge: Cambridge University Press. URL: http://www.astro.caltech.edu/ george/ay21/ readings/guth. pdf

Hawking S. 2001. A Brief History of Time. From the Big Bang to Black Holes. St. Petersburg: Amfora. In Russian (Хокинг С. Краткая история времени. От большого взрыва до черных дыр. СПб.: Амфора). 
Levin A. 2010. A Trillion Years before the Big Bang. Popular Mechanics 6. URL: http://elementy.ru/lib/431131?page design=print. In Russian (Левин А. За триллион лет до Большого взрыва. Популярная механика 6).

Mukhanov V. F., and Orlova O. 2006. Big Bang Became a Victim of Inflation. In Russian (Муханов В. Ф., Орлова О. 2006. Большой взрыв стал жертвой инфляции. URL: http://polit.ru/article/2006/12/19/vzryv).

Postnov K. A. 2001. Lectures on General Astrophysics for Physicists. Moscow: Moscow State University. In Russian (Постнов К. А. Лекции по общей астрофизике для физиков. М.: Изд-во МГУ. URL: http://www.astronet.ru/db/ msg/1170612/index.html).

Rubin S. 2004. The World Born out of Nothing. Vokrug Sveta 2(2761): 56-65. In Russian (Рубин С. 2004. Мир, рожденный из ничего. Вокруг света 2(2761): 56-65).

Sazhin M. V. 2002. Modern Cosmology in Popular Presentation. Moscow: URSS. In Russian (Сажин М. B. 2002. Современная космология в популярном изложении. М.: Едиториал УРСС).

Vilenkin, A. 2006. Many Worlds in One: The Search for Other Universes. New York: Nill and Wangs.

Vilenkin A. 2010. The World of Many Worlds. Physicists in Search for Other Universes. Moscow: Astrel. In Russian (Виленкин А. Мир многих миров. Физики в поисках иных вселенных. М.: Астрель).

Wood B. 2018. Imagining the Unimaginable: Narratives of the Big Bang Time, Space, Matter, Energy. Journal of Big History 2(1): 1-13. URL: http://dx.doi.org/10.22339/jbh.v2i1.2251. 
